\title{
14 \\ The Optimal Utilization of Multi-Service SCP
}

\author{
Tang Haitao and Olli Simula \\ Laboratory of Computer and Information Science \\ Helsinki University of Technology \\ Rakentajanaukio $2 C$ \\ 02150 ESPOO, FINLAND \\ Tel: $+35804513288,+35804513271$ \\ Fax: +35804513277, +35804513277 \\ E-mail: Haitao.Tang@hut.fi, Olli.Simula@hut.fi
}

\begin{abstract}
This paper introduces one method for the optimal utilization of the SCP which offers several kinds of services at the same time. There are three issues related to this method, i.e. the simulation of the traffic for the services, the approximation of the service-related system capacities, and the adaptation for the environment which is constructed mainly by the traffic for the different services. The adaptation (called SOM-D) is the main part of this paper. It consists of using Self-Organizing Feature Map to encode the time-varying environment and using one adaptive gap decoder to get the optimal gap sequences. Simulation is made to test the method. The results show that the system performance can be significantly improved by this adaptive method.
\end{abstract}

\section{INTRODUCTION}

Many system design problems are essentially resource management problems which can be done more efficiently if designer knows more about the on-going resource requirements. However, searching for the distributions of the on-going resource requirements is already very difficult sometimes, even if it may be possible. Then, approximation and adaptation are the logical results for dealing this kind of problems. It means that the suitable approximations of resource requirements and case-dependent system capacities as well as the needed adaptation method should be found. Based on those considerations, one method to increase the system 
efficiency of a Service Control Point (SCP) and its Quality of Service (QoS) is introduced in this paper.

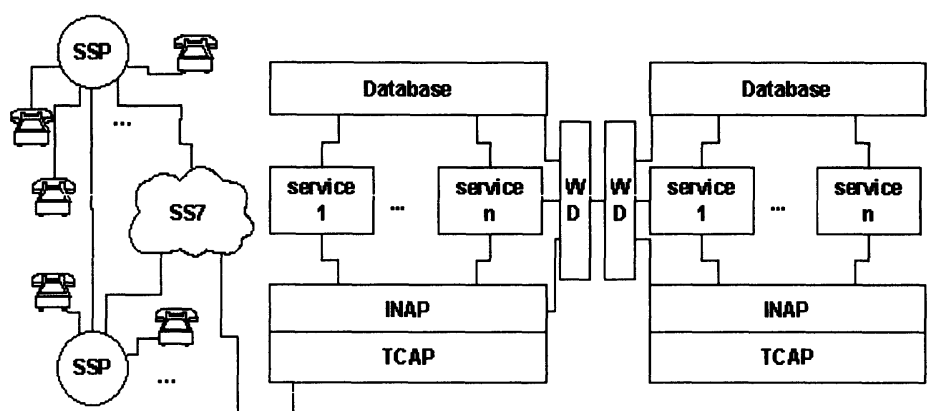

Figure 1 The Service Control Point

Figure 1 shows a SCP which supports several kinds of services at the same time. The WD (watchdog) in Figure 1 is the local manager of the SCP. It is introduced in [9]. The SCP is implemented as distributed processes inside a host computer. It is clear that the SCP is a timesharing system.

There are many ways to model the SCP system. One may model it through the arrival of packets and the way of handling them (modeling at packet level). However, this way may not be quite suitable for the case of Figure 1. One reason is that it is very complex to model the sources (the caller) on the base of packet. Even though a call for certain service is closely related to certain sequences of packets, the caller (human being) will greatly affect system service rate by the delay of his manual operation. If one models the system through the arrival of calls and the way of handling them, the delay of human operation will be easier to trace and put into the account of modeling. Another reason is that complete tracing of packets seems an expensive way for the case of Figure 1 even if it is possible, which will use too much system resources so that the system capacity for real services is significantly reduced.

It is quite reasonable to use a straight forward way, modeling the system through the arrival of calls and the way of handling them (modeling at call level). This uses much less system resources than the packet level modeling, even through the call start, end, and service type of a call are needed to be checked from packet level. The parameters needed for modeling are also easier to measure in real situation. Then, the SCP system without any adaptive mechanism is modeled on the base of call as Figure 2.

Suppose that there are $n$ different kinds of services in the system, named as service 1, service $2, \ldots$, and service $\mathrm{n}$, in Figure 2. $g_{h_{1}}, g_{h_{2}}, \ldots$, and $g_{h n}$ are fixed gaps in terms of maximal acceptable calls in unit time $T$ for corresponding service. $\lambda_{1}, \lambda_{2}, \ldots$, and $\lambda_{n}$ are the total numbers of calls for corresponding service and arriving in unit time $T$; the numbers include dropped calls. The 
system service rates for service 1 , service $2, \ldots$, and service $\mathrm{n}$ are noted as $\mu_{h 1}, \mu_{h 2}, \ldots$, and $\mu_{h n}$, respectively. $\mu_{c}$ is system capacity.

Since the system in Figure 2 uses fixed gaps, the optimal use of the system cannot be reached or the optimum could be reached for certain situation but not keeping the optimum when situation is changing. Therefore, using fixed gaps may not be a good way.

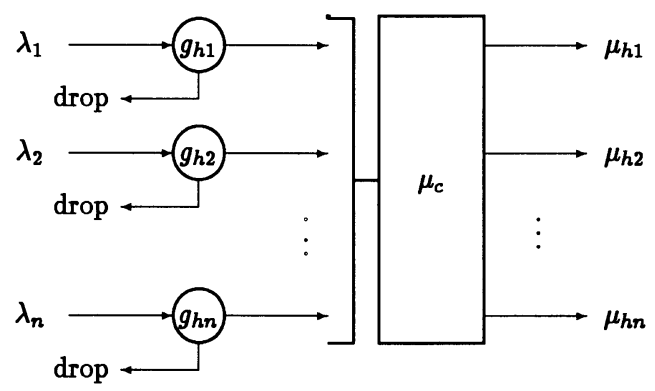

Figure 2 The model of the SCP without adaptation

If one tests the number of arriving calls for certain service in every unit time $T$, he can find that its distribution may not be stable. However, it is reasonable to believe $[1,2,10]$, when the test is kept for days or even weeks or longer $\left(T_{t}\right)$, certain relations can be found. The relations may be approximations of certain statistical relations. Figure 3 shows our simulation of calls for three different services in a day. Then, certain relations among the three different service calls are generated when the services are implemented in same host computer. In fact, those principles hold for any number of different services in the system.

By comparing the results from different services in Figure 3, one may be sure that, if he is able to encode the multi-dimensional relations into finite states, certain optimum can be reached through suitable decoding. The encoding and decoding processes are considered as an approximation of real situations. When situations change, the code book will change in order to adapt to the new situations and then, new optimum will be reached through the decoding. If the changes of situations are slow when compared to the updating rate of the code book, the newly updated code book can be used to approximate the situations in the coming period. In turn, the approximated optimum can be reached for the coming period. The optimum mentioned here is the global optimum in the period $\left(T_{t}\right)$ between two successive code book updates.

The SCP system with adaptive gaps can be modeled as shown in Figure 4, which is also on the base of call like that in Figure 2; In Figure $4, g_{1}, g_{2}, \ldots$, and $g_{n}$ are adaptive gaps which are the outputs of the gap-adapting block; $\mu_{1}, \mu_{2}, \ldots$, and $\mu_{n}$ are service rates for service 1 , service $2, \ldots$, and service $n$, which are affected by the adaptive gaps. 


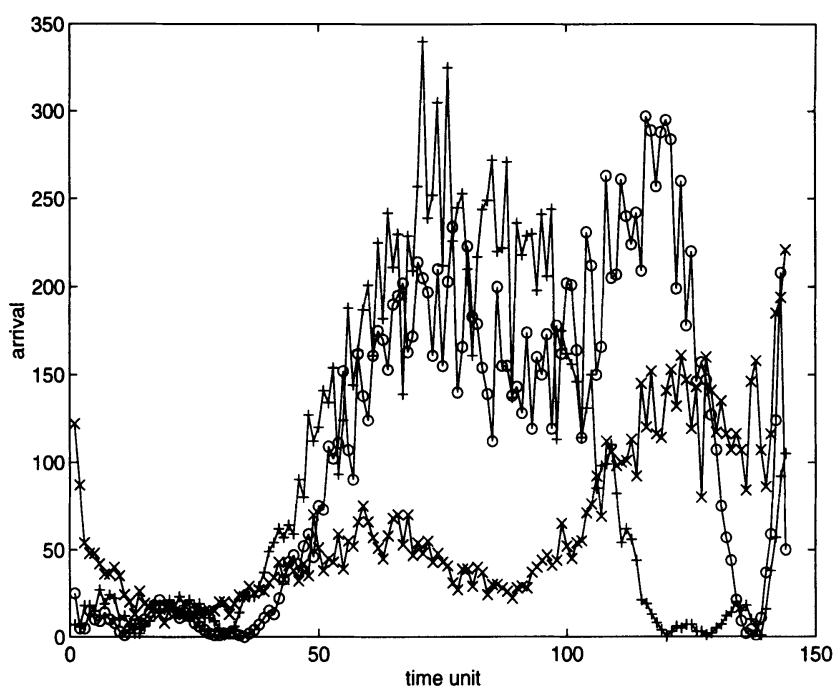

Figure 3 The simulated traffic for three different services in a day. X-axis is the unit time in a day and each unit time is ten minutes. Y-axis is the arrivals of calls for services. Line '-+-' is for service 1 , line '-x-" for service 2 , and line '-0-' for service 3.

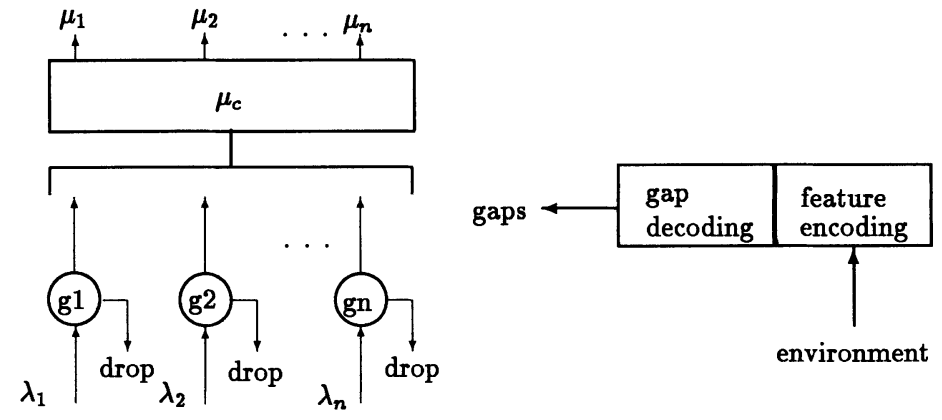

Figure 4 The model of the SCP with gap adaptation

\section{THE ADAPTATION THROUGH SOM-D}

In this section, there are three issues to be introduced: a kind of system capacity approximation, the adaptive encoding of the call environments through Self-Organizing Feature Map [3, 4], and the decoding for adaptive gaps of the SCP. The process of encoding and decoding together is called as SOM-D. 


\subsection{System Capacity}

The system capacity (maximal number of acceptable calls in unit time $T$ ) for certain service is decided by the embodied capacity of the system and its service environments. In principle, it should be smaller than the embodied capacity of the system. Since we are interested in the system capacities for certain services, the latter will be simply called system capacity in the following context.

There could be many ways to measure the system capacity, e.g., from call level or packet level, etc. Even though each of the methods is approximative, some method could get the result which is closer to the real system capacity if certain conditions are considered.

The results from call level methods may not be good for a SCP system because the human behavior affects the call for service and it is changing with time, location, culture, etc. Therefore, for that case, the results may not be reliable and could be very expensive even if it is possible to measure through those methods. There are a lot of limitations which make those methods not workable practically.

Comparably, the methods from packet level seems more reliable for the results and more practicable. It is known that certain call for certain service is implemented by certain sequences of packets if looking from packet level. The sequences could have some change, but the results could not be possible to change too much if considering that, with very high probability, the call either is accepted and correctly processed or is refused back (drop out).

More reliable results can be obtained from the worst cases-there is no extra time delay existing, i.e. when caller SSP (Service Switching Point) receives some packet from the SCP, it can immediately return related packet to the SCP if needed. Certainly, the results are usually strict and have some distance from the system capacity. But, it is one of the most deterministic results which people could get.

One good side effect of using the strict capacity to approximate the system capacity is that some amount of system capacity will be not used. If the idle part is not too big (say, $<25 \%$ of the system capacity), it is good to be a safeguard for possible system congestion. The system delay can be reasonably low when accepting calls under the limit of those strict capacities. The experiences from real situations give people the confidence, it is better to put some safeguard for using the system capacity (i.e. let the system utilization factor $\rho<1$ ).

One usable complement for the possible "too strict" could be the strict capacity updating; that is, when real call traffic exceeds the bound of the strict capacity, increase the strict capacity bound by a factor of $\gamma, 1<\gamma<2$ and stop increasing before global service time delay starts to increase significantly. The newly found strict capacity could be set at 0.8 or 0.9 of the turning point. This method can decrease the distance between the strict capacity and the system capacity if needed.

Here is the way of measuring the strict capacities. It is to measure the strict capacities on the base that system serves one kind of service at a time. Through this way, the strict capacity for system to serve a certain kind of service alone can be found. The algorithm is the following: 
(a) Find one single host computer for the SSP simulator which is not the SCP host and whose processing speed should be much faster than that for the SCP. If not much faster, the results should be adjusted on case-dependent base.

Let $i=1$.

(b) Choose the kind of service $i$ in the SCP. The SSP simulator creates calls for the service and sends them to the SCP as well as keeps the traffic in a way, if one call is served by the SCP, a new call is sent to the SCP immediately. Simulator keeps this process by increasing $m$ until the global service time of the system starts to increase significantly (the start of congestion). Then, hold this turning point (a little bit smaller than it) for the results measuring.

Continue the measuring for $\mathrm{L}$ unit time $T$. Let $\lambda_{a i j}$ be the accepted calls in $j^{\text {th }}$ unit time $T$ and $\lambda_{a i}^{*}$ be the maximal acceptable calls in unit time $T$ for service $i$. Then,

$$
\lambda_{a i}^{*} \approx \frac{1}{L} \sum_{j=1}^{L} \lambda_{a i j}
$$

We know that the above test is on the base of "one out and then one in"'. Let $\mu_{i}^{*}$ be the system capacity for service i. If $L$ is large enough, it is reasonable to assume,

$$
\mu_{i}^{*} \approx \lambda_{a i}^{*}
$$

Let $i=i+1$, if $i>n$, end (b); else iterate (b).

Now, the $n$ strict capacities or maximal acceptable calls for the $n$ services by the SCP are found. It is reasonable to say,

$$
\mu_{i}^{*} \approx \mu_{c_{i},} \text { (service i) }(i=1,2, \ldots n)
$$

$\mu_{c_{i}}$ is the real system capacity for service i. Let $\mu_{\alpha}^{*} \approx \min _{i}\left\{\mu_{i}^{*}\right\}$ and $\alpha_{i}=\frac{\mu_{i}^{*}}{\mu_{\alpha}^{*}}$. Then,

$$
\left.\mu_{c_{i}} \approx \alpha_{i} \mu_{\alpha}^{*} \text { (service } i\right)(i=1,2, \ldots n)
$$

Thus, one may think that the SCP consists of the basic servers and each of them has $\mu_{\alpha}^{*}$ service rate.

We know that the approximated system utilization has to be,

$$
\sum_{i=1}^{n} \frac{g_{i}}{\mu_{c_{i}}} \leq 1
$$

Therefore, the upper bound for the maximal acceptable calls in unit time $T$ can be, 


$$
\sum_{i=1}^{n} \frac{g_{i}}{\lambda_{a i}^{*}} \approx 1
$$

Because there are quite many possible solutions for (6), one should be able to find the solution which is the closest match of the environment at that time. The wanted solution can be found through (12) quickly. The details will be introduced in Section 2.5.

\subsection{On Optimums of the System}

Making Figure 4 as an example, in principle, one could find different system optimums from different cost functions which are built for different purposes, e.g., to get maximal global call throughput, to get maximal global call billing, or both of them, etc.

Suppose that one could find the following cost function;

$$
\mathbf{E}=\mu \mathbf{a}^{\mathrm{T}}+\mathbf{Q \mathbf { b } ^ { \mathbf { T } }}
$$

service rate: $\mu=\left(\mu_{1}, \mu_{2}, \ldots, \mu_{n}\right)$

service cost: $\mathbf{a}=\left(a_{1}, a_{2}, \ldots, a_{n}\right)$

staying cost: $\mathbf{b}=\left(b_{1}, b_{2}, \ldots, b_{n}\right)$

Then, try to find $\min _{\mu} \mathbf{E}$ through (if it exists),

$$
\text { average number: } \mathbf{Q}=\left(q_{1}, q_{2}, \ldots, q_{n}\right)
$$

$$
\frac{\partial \mathbf{E}}{\partial \mu}=\frac{\partial\left(\mu \mathbf{a}^{\mathbf{T}}+\mathbf{Q b}^{\mathbf{T}}\right)}{\partial \mu}=0
$$

One may find that it is very hard (sometime even not possible) to find (7) or (8) from real systems as above. Its approximation may be very difficult to get, too. Thus, for the SCP case, directly trying this way is not suitable.

It seems that, if one can find certain approximation of the probability space of the system with its environments, the global optimums of the system could be approximated through certain adaptive method. The method should be directly based on the measured data from system. It makes the approximation and real time processing easier.

\subsection{On SOM}

The self-organizing feature map (SOM) is the Kohonen model [3]. It is a kind of unsupervised learning neural network and, in reality, it belongs to the class of vector coding algorithms [4]. It can learn the environment constructed by input vectors through itself.

The self-organizing feature mapping $\Phi$ has some usable properties as the following [4], (a) approximation of the input space, (b) topological ordering which relates to input patterns, (c) density matching which reflects the variations in the statistics of the input distribution. The property (a) and (c) are especially useful for the approximation of the probability space in the SCP case. For the details of SOM, please read the related materials in [3, 4]. 
As an example, a two-dimensional lattice of neurons of SOM is shown in Figure 5.

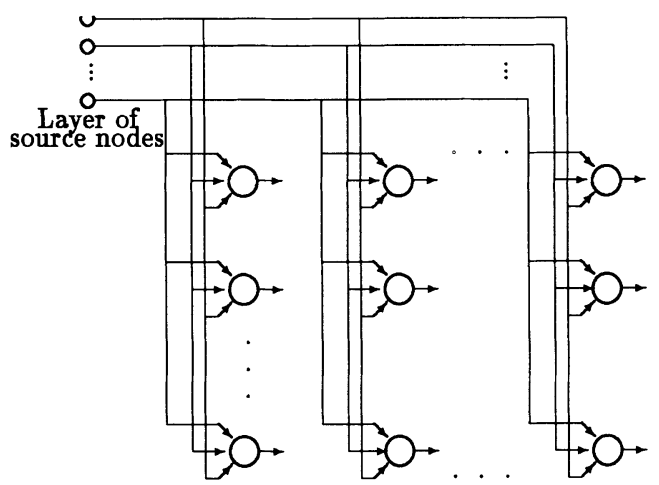

Figure 5 One example of two-dimensional SOM

The summary of SOM algorithm is listed as the following [4],

(A) Initialization. Choose random values for the initial weight vectors $\mathbf{m}_{j}(0)$. The only restriction here is that the $\mathbf{m}_{j}(0)$ be different for $j=1,2, \ldots, N$, where $\mathrm{N}$ is the number of neurons in the lattice. It may be desirable to keep the magnitude of the weights small.

(B) Sampling. Draw a sample $\mathbf{x}$ from the input distribution with a certain probability; the vector $\mathbf{X}$ is the sensory signal.

(C) Similarity Matching. Find the best-matching (winning) neuron $i(\mathbf{x})$ at time $\mathrm{n}$, using the minimum-distance Euclidean criterion:

$$
\mathrm{i}(\mathbf{x})=\arg \min _{j}\left\|\mathbf{x}(\mathrm{n})-\mathbf{m}_{j}\right\|, j=1,2, \ldots, N
$$

(D) Updating. Adjust the synatic weight vectors of all neurons, using the update formula,

$$
\mathbf{m}_{j}(n+1)=\left\{\begin{array}{l}
\mathbf{m}_{j}(n)+\eta(n)\left[\mathbf{x}(n)-\mathbf{m}_{j}(n)\right], j \in \Lambda_{i(x)}(n) \\
\mathbf{m}_{j} \text { otherwise }
\end{array}\right.
$$

where $\eta(n)$ is the learning-rate parameter, and $\Lambda_{i(x)}(n)$ is the neighborhood function centered around the winning neuron $\mathrm{i}(\mathbf{x})$; both of them are varied dynamically during learning for best results.

(E) Continuation. Continue with step B until no noticeable changes in the feature map are observed. 


\section{$2.4 \quad$ Encode Calls through SOM}

Suppose that the results in Figure 3 are obtained through period $T_{t}$. Since customer behavior and situation are comparably "stable", it is reasonable to believe that the results tells the relations of global situations in the period $T_{t}$. If $T_{t}\left(1 / T_{t}\right.$ is updating rate of the code book) is comparably small and environment change is slower than the updating rate, it is also reasonable to believe that the newly updated code book can be used for the coming period, $\left(T_{t}+1\right)$, in the way of "weekday for weekday and weekend for weekend, etc.". The environment changes in the coming period can be approximated as the quantitative changes of calls of all the unit time $T$ in $T_{t}$. The call change can be quantized with a fuzzy coefficient $\beta(9)$ and the training set $S_{t}$ is constructed through (10). The $t_{h}, t_{d}$, and $M$ in (10) are the time in a day, the time in a week, and the total number of sample vectors used. $\lambda_{1 j}, \lambda_{2 j}, \ldots$, and $\lambda_{n j}$ are the measured call arrivals for service 1 , service $2, \ldots$, and service $n$, respectively, in the $j$ th unit time $T$.

$\beta$ (finite set of positive values

$$
S_{t}=\left\{\left(\left(\beta \lambda_{1 j}, \beta \lambda_{2 j}, \ldots, \beta \lambda_{n j}\right), t_{h}, t_{d}\right) \mid j=1,2, \ldots, M(\beta\}\right.
$$

The self-organizing feature map is constructed by two-dimensional lattice of neurons, e.g., in size of $64 \times 64$. It is properly initialized and then trained by the input sequence of the vectors which are randomly picked from the set $S_{t}$ until the SOM reaches convergence phase. Then, the SOM can been seen as a code book for the environment. The environment in $T_{t}$ is coded into the weights of the neurons. It is the optimal approximation of the environment in the sense of probability.

Now, the necessary conditions for constructing suitable decoding mechanism has been met, which produces the adaptive gaps. The gaps are optimally matching the traffic in global sense for the period $T_{t}$.

\subsection{Decode for Adaptive Gaps}

The idea of the SOM-D adaptation is shown in Figure 6. The algorithm of the decoding for the adaptive gaps is the following:

Let $\mathbf{m}_{i}$ be the weight vector of neuron $\mathrm{i}, \mathbf{m}_{i}=\left(m_{1 i}, m_{2 i}, \ldots, m_{(n+2) i}\right)$ and let $\mathbf{v}_{j}$ be one input vector from the environment in period $\left(T_{t}+1\right), \mathbf{v}_{j}=\left(\lambda_{1}, \lambda_{2}, \ldots, \lambda_{n}, t_{h}, t_{d}\right)$. Let $\mathbf{w}$ be the set which holds all the weights of the SOM.

(a) Input $\mathbf{v}_{j}$ into the SOM and then find the weight, $\mathbf{m}_{j}=\left(m_{1 j}, m_{2 j}, \ldots, m_{(n+2) j}\right)$, which meets,

$$
\min _{\mathbf{w}}\left\|\mathbf{v}_{j}-\mathbf{m}_{j}\right\|
$$

(b) decode them into the adaptive gaps for the unit time $T^{\text {tith }}$ by, 


$$
g_{k}=\operatorname{round}_{u p}\left(\lambda_{a k}^{*}\left(\frac{m_{k j}}{\sum_{l=1}^{n} m_{l j}}\right)\right), k=1,2, \ldots, n
$$

(c) use these gaps for next unit time $T^{(j+1) t h}$.

In fact, it is not needed to care the outlooking of the SOM for the adaptive coding and decoding. It is good for no need of extra-intervention.

The decoding in (12) has several characters. It constructs the strict upper bound by its gaps from decoding (see (6)) and optimally matches the environment in the sense of probability by its gaps. It gives optimal throughput and also prevents possible system congestion.

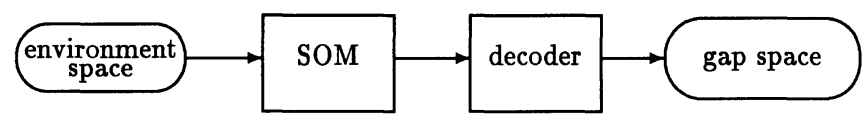

Figure 6 The principle of the adaptation method

\subsection{SOM-D with Priority}

The above SOM-D treats any service kind with equality in the sense of the fair share of system capacity. However, for some cases, e.g., emergency service, important televoting, etc., one has to decrease the load from other services by force if needed. It means that services should be tagged with different priorities for those cases. It also means that a compromise is required between the optimum and the wanted unfairness. This problem can be solved through the reconstruction of the gap space, etc. This part is not, however, introduced in this paper.

\section{SIMULATION RESULTS}

For simulation, three different kinds of simulated services are tested in our simulation SCP implemented in PC 486 with Linux OS. The traffic of ten working days is simulated for each service. Figure 3 shows one day's traffic. The first five days' traffic is used for training the SOM-D. Then, the next five days' traffic is used for the performance simulation of the SCP with SOM-D. One software packet is used for the SOM [6].

The SOM is updated every five days (omiting weekends at this moment). If the overall traffic distributions change slower than map update does, SOM-D works well. Otherwise, the fixed gaps can be used temporarily until SOM-D matches the call environment. The adaptive gaps are updated every ten minutes. Figure 7 shows the sequences of gaps in one day. One can see that the three sequences of gaps follow the potentials of the simulated traffic and compromise with each other in order to hold the upper bound of the maximal acceptable calls in unit time $\mathrm{T}$ for the call environment. The upper bound also prevents system from possible congestion. 


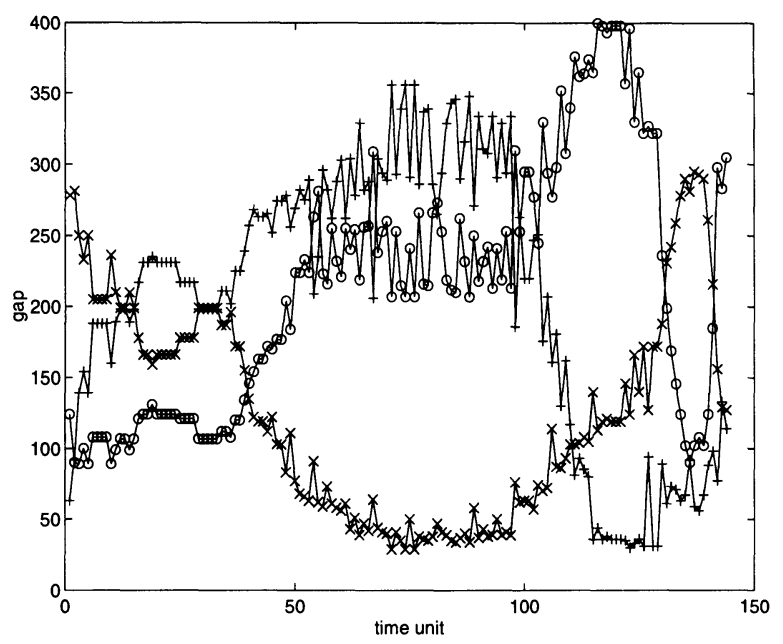

Figure 7 The sequences of the adaptive gaps for different services in a day. $\mathrm{X}$-axis is the unit time in a day and each unit time is ten minutes. The gaps are updated every unit time. Y-axis is the maximal acceptable calls for the services at each unit time. Line '-+-' is for service 1 , line '- $\mathrm{x}$-' for service 2 , and line '-o-' for service 3.

For performance simulation, different levels of traffic are simulated. Since no congestion can happen under the SOM-D, reasonably low system delay is guaranteed. Figure 8 shows the throughput improvement by using the adaptive gaps. For a better understanding of the improvement, the system delay, when the SCP has no any congestion control mechanism, is simulated with different traffic levels; it is also shown in Figure 8.

From Figure 3, one can find that the traffic in a day are unbalanced. It means that some amount of system resource is not used in certain times of the day, even through the system can be very busy in other times of the day. This kind of unbalance is caused by customers. Similar unbalance has been seen in many papers $[1,2,5,10]$, etc. It is very clear that one cannot count on it to improve the system performance. However, it is found that certain unbalances exist among the traffic levels from different service calls most of the time. The unbalances among the traffic levels offer us the opportunity to improve the system performance.

From Figure 8, one can find that, if using fixed gaps, the system throughput decreases far before the onset of the potential system congestion (relative traffic level 2.1 to 2.5 , if happened). When using adaptive gaps, the system throughput is improved, the throughput band is expanded and it is closer to the onset of potential system congestion. When traffic is small, the improvement is small (the only possibility). The improvement increases with the increase of traffic until certain point. Then the improvement keeps a certain value (about $20 \%$ in Figure 8 ) and starts to decrease slowly. There may be two reasons that the improvement dose not increase linearly with the traffic. One is that there are still remaining differences between the approximation from SOM-D and the call environment. The other is that, when traffic in- 
creases after certain level, more and more calls have to be dropped in order to keep system from congestion. Thus, in fact, SOM-D functions as both optimal system utilization and flow control mechanisms.

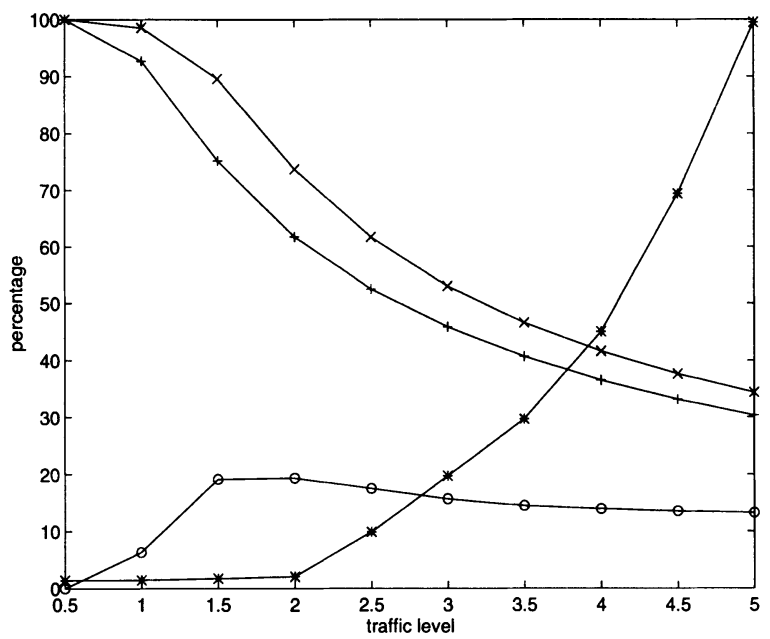

Figure 8 The performance improvement for the simulated traffic through SOM-D. X-axis is relative traffic level by comparing with certain amplitude of the simulated traffic. Y-axis at left side is percentage. For line '- $\mathrm{x}-$ ' and line '-+-', the Y-axis means the percentage of real acceptable calls by comparing to the arrivals of calls for services (we call the percentage as effective throughput or, simply, throughput. It is a little bit different from the traditional throughput measurement). Line '- $\mathrm{x}-$ " is the throughput by using SOM-D. Line "-+-' is the throughput by using fixed gaps. Line '- 0 -" is the throughput improvement by comparing the throughput of SOM-D with that of fixed gaps. The y-axis at right side means system delay in second. Line "-

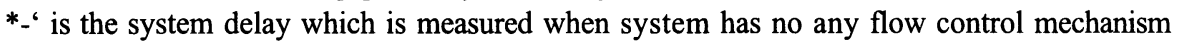
used. We use it to help the analysis of the other results only.

In addition, there is still some difference between the improved throughput band and the onset of potential congestion (if no flow control mechanism used). The main factors for this difference are the unbalance of the traffic along the time and the distribution among the traffic levels. These two factors are decided by call environment (or the customers). It means that the call environment decides the upper bound for performance improvements. What we can do is to push the system performance closer to this upper bound. 


\section{CONCLUSION}

Usually, when designing certain telecommunication systems, more attention is paid to using more powerful components than to the optimal operation of the system in case that the system performance is not good enough. We do know that the system capacity has to be increased in order to increase its performance under some situations. However, we have many experiences that, even if the average system utilization is very low, the performance of the system is sometimes starting to degrade very fast. Indeed, there may be many factors which cause this situation. System configuration, both static and dynamic, can be one of them. It means that only increasing system capacity may not be enough or so efficient and feasible.

In this study, one method for measuring service-dependent system capacity and one adaptive gap updating mechanism are introduced. The performance improvement is also proved through the simulation. In the developing of the methods, the capacity of SS7 is supposed to be sufficient for simplicity in the analysis.

From the simulation, it is demonstrated that system throughput can be improved and, at the same time, low system delay can be guaranteed as well as potential system congestion can be prevented; all these are done without necessarily using more powerful components. We hope this study can attract some attentions into the optimal management of some telecommunication systems, too.

We know that to upgrade the performance is also part of the efforts to increase Quality of Service (QoS) which is visible to customer. Then, the use of SOM-D for adaptive gaps helps to upgrade the serviceability performance of the SCP $[7,8]$.

Indeed, the SOM-D method adds extra load to the system, even if it is comparably small. Fortunately, in most cases, SCP is implemented with double systems, one active and the other standby at the time, in order to guarantee its service availability [9]. The processing load from SOM-D can be put into the standby system. The active system executes only gap updating. Other needed operations are the same as in the SCP without SOM-D. As a result, it means that hardly any extra load is added into the active part of the SCP, and thus, nearly no extra overhead is added.

\section{ACKNOWLEDGMENT}

We are grateful to Tapani Karttunen (Telecom Research Center/Telecom Finland Ltd) for his helpful comments on this paper and the valuable information from the cooperation between us and his group. We also like to thank Kimmo E. E. Raatikainen (Dept. of Computer Science/Helsinki University) for his valuable comments on the paper.

\section{REFERENCES}

[1] R. I. Wilkinson, "Theories for toll traffic engineering in the U. S. A.," Bell Syst. Tech. J. vol. 35, no. 2, pp.421-514, Mar. 1956. 
[2] W. S. Hayward and J. P. Moreland, "Total network data system: Theoretical and engineering foundations, “ Bell Syst. Tech. J., vol. 62, no. 7, pp. 2183 - 2207, Sept. 1983.

[3] T. Kohonen, "Self-Organizing Maps" Springer-Verlag, 1995.

[4] Simon Haykin, "Neural Networks," Macmillan College Publishing Company, Inc., pp. $397-434,1994$.

[5] Henry J. Fouler and Will E. Leland, "Local Area Network Traffic Characteristics, with Implications for Broadband Network Congestion Management," IEEE J. on Selected Areas in Communications, vol. 9, no. 7, pp. 1139 - 1149, Sept. 1991.

[6] Kohonen T., Hynninen J., Kangas J., and Laaksonen J., "SOM-PAK, The Self- Organizing Map Program Package, ver. 3.1," Mar. 1995.

[7] E.7IN1. ITU-SG2, "Draft Recommendation".

[8] CCITT Recommendations Q.1200 series, Intelligent Networks, Geneva, Switzerland (1992).

[9] Tang Haitao and Karkkainen Esa, "The Local Management for A Service Control Point," IFIP TC6 Workshop on Intelligent Networks, vol. I, pp. 45 - 55, Aug. 1994.

[10] EURESCOM P308, "Methods and Specifications for Tools to Dimension Intelligent Networks, Deliverable 2, IN Service Model and Traffic Performance Analysis", Volume 1 of 2: Main Part, March 1995.

\section{BIOGRAPHY}

Tang, Haitao was born in Kunming, China, Nov. 1961. He received the Master's Degree from Nanjing Institute of Telecommunications in 1986. Since then, he worked at Beijing Data Communication Co. as a researcher for designing network equipment until 1992. From 1993 to 1995, he worked as a researcher of Intelligent Networks at Telecom Finland Ltd. Currently, he is a researcher and a senior postgraduate student working towards his Ph.D. at the Laboratory of Computer and Information Science in Helsinki University of Technology. His current research interest is on the adaptive methods for telecommunication systems.

Olli Simula was born in Helsinki, Finland, on July 22, 1948. He has received the degree of Doctor of Technology from Helsinki University of Technology in computer science in 1979. Since 1974 Dr. Simula has been with the Laboratory of Computer and Information Science at the Helsinki University of Technology, being currently Associate Professor of Computer Science. During 1977-78 he worked one year as a Research Fellow at the Delft University of Technology, Delft, the Netherlands. His research interests include digital signal processing and computer architectures for signal processing as well as neural networks and their applications in process monitoring and analysis. 